\title{
Progress on Nonlinear-Wave-Forced Sediment Transport Simulation
}

\author{
Richard W. Gilbert, Emily A. Zedler, Stéphan T. Grilli, and Robert L. Street
}

\begin{abstract}
In this paper, we report on the use of a numerical wave tank (NWT), based on fully nonlinear potential flow (FNPF) equations, in driving simulations of flow and sediment transport around partially buried obstacles. The suspended sediment transport is modeled in the near-field in a Navier-Stokes (NS) model using an immersed-boundary method and an attached sediment transport simulation module. Turbulence is represented by large eddy simulation (LES). The NWT is based on a higher order boundary element method (BEM), with an explicit second-order time stepping. Hence, only the NWT boundary is discretized. The solution for the velocity potential and its derivatives along the boundary is obtained in the BEM, which subsequently provides a solution at any required internal point within the domain. At initial time, the NS-LES model domain is initialized with the 3-D velocity field provided by the NWT and driven for later time by the pressure gradient field obtained in the NWT. Incident wave fields, as specified in the NWT to drive sediment transport, can be arbitrary. Applications are presented here for single frequency waves, such as produced by a harmonic piston wavemaker in the laboratory, and modulated frequency wave groups. The feasibility of coupling the irrotational flow and NS solutions is demonstrated.
\end{abstract}

Index Terms-Boundary element method (BEM), coastal processes, computational fluid dynamics, model coupling, nonlinear waves, sediment transport.

\section{INTRODUCTION}

$\mathbf{I}$ $\mathrm{N}$ this paper, we report on our progress towards the modeling of wave-induced flow and suspended sediment transport around partially buried cylindrical obstacles, using a coupled wave-hydrodynamic-sediment transport model. While most similar work to date has usually been based on specifying a simple oscillatory flow to force the sediment transport [1]-[4],

Manuscript received March 31, 2005; revised April 4, 2006; accepted June 29, 2006. This work was supported by the U.S. Office of Naval Research (ONR) Coastal Dynamics Program under Grant N00014-00-1-0440 and the ONR Coastal Geosciences Division Mine Burial Burial Program under Grant N00014-05-1-0068 (code 321CG)

Guest Editor: M. D. Richardson.

R. W. Gilbert was with the Department of Ocean Engineering, University of Rhode Island, Narragansett, RI 02882 USA. He is now with the McLaren Engineering Group, West Nyack, NY 10994 USA (e-mail: rgilbert@mgmclaren. com).

E. A. Zedler was with the Environmental Fluid Mechanics Laboratory, Department of Civil and Environmental Engineering, Stanford University, Stanford, CA 94305 USA. She is now with the Santa Clara Valley Water District, San Jose, CA 95118 USA (e-mail: zedler@gmail.com).

S. T. Grilli is with the Department of Ocean Engineering, University of Rhode Island, Narragansett, RI 02882 USA (e-mail: grilli@oce.uri.edu).

R. L. Street is with the Environmental Fluid Mechanics Laboratory, Department of Civil and Environmental Engineering, Stanford University, Stanford, CA 94305 USA.

Color versions of one or more of the figures in this paper are available online at http://ieeexplore.ieee.org.

Digital Object Identifier 10.1109/JOE.2007.890979 here, we use the more realistic background wave velocity field created by fully nonlinear waves, both over flat bottom and shoaling over slopes. Earlier research indeed shows that nonlinear effects in shoaling wave fields lead to asymmetries in both wave shape and near-bottom currents that strongly affect sediment transport (e.g., [5]-[9]) and the resulting burial or unburial of obstacles located on the bottom.

When approaching shore, due to refraction, shoaling ocean swells often become locally 2-D in the cross-shore direction. A 2-D waveform is thus a good approximation for simulating wave-induced sediment transport in an essentially cross-shore direction (e.g., Fig. 1). Recent laboratory experiments performed in a wave tank with moving bottom at the Arizona State University (ASU, Tempe, AZ, [5], [6], [8]-[10]) show that small scale disturbances in the bottom topography, as compared to the wavelength, such as sand ripples or small partially buried obstacles, usually affect flow velocities only within 2-3 significant diameters of the object and have negligible effects on the waveform. This allows us to simulate the 2-D wave "far-fields," to provide background wave velocities for driving the 3-D turbulent flow that occurs around bottom obstacles, in the "near-field."

We model far-field waves in a 2-D numerical wave tank (NWT), solving fully nonlinear potential flow (FNPF) equations, based on a higher order boundary element method (BEM) and an explicit time stepping [7]-[9], [11]-[14]. The potential flow approximation (i.e., irrotational motion of an inviscid, incompressible fluid) used in this NWT has been shown in earlier work to be very accurate for simulating both the shape and velocities of shoaling waves, as compared to laboratory measurements (e.g., [7], [8], and [15]-[17]). Figs. 1 and 2, for instance, show the setup, and then, the results of a recent comparison of simulations in this 2-D-NWT, with laboratory experiments at ASU [8], [9]. We see that both the simulated period-averaged wave elevation close to breaking and the near-bottom horizontal velocity are in good agreement with measurements. Discrepancies of only a few percent before breaking are typical.

Here, the 2-D-NWT is similarly used to simulate wave generation, propagation over a flat bottom, and transformations (i.e., shoaling) over a sloping bottom topography, and corresponding wave-induced internal velocities and pressure fields. These serve as both initialization and background forcing, as a function of time, for a near-field 3-D Navier-Stokes (NS) hydrodynamic model. At this time, no feedback of NS model results into the NWT is implemented. This so-called "weak model coupling" approach was successfully applied in earlier work to the modeling of shoaling, breaking, and postbreaking waves, by using a 2-D- or a 3-D-FNPF-NWT to initialize and 


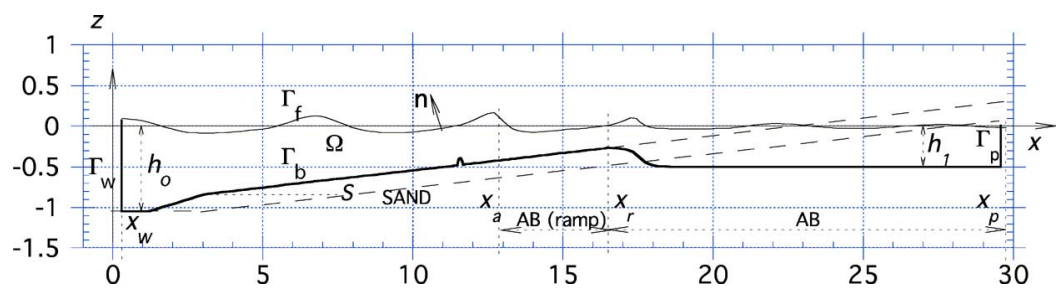

Fig. 1. Sketch of the 2-D-NWT setup for computations of wave shoaling and breaking over a sandy slope $S=1: 24$. Note that AB is absorbing beach for $x \geq x_{a}$; a piston wavemaker is located at $x=x_{w}$; and $(--)$ indicates the position of the sand layer in the laboratory wave tank used at ASU, for which dimensions are in meters [8], [9]. In these experiments, there was a $8.4-\mathrm{cm}$ radius, $50 \%$ buried cylinder at $x=12.8 \mathrm{~m}$.
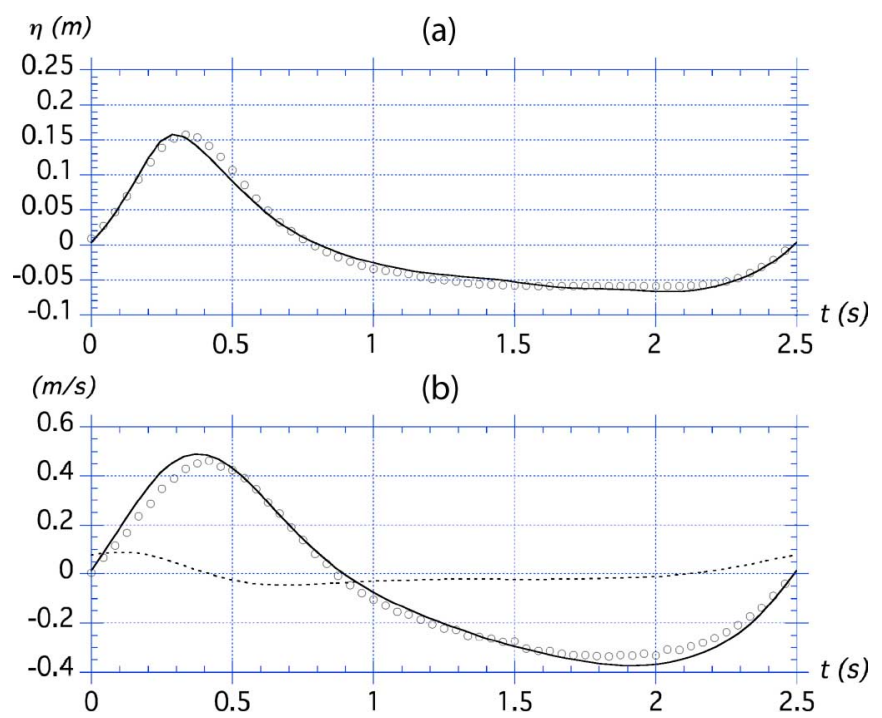

Fig. 2. Example of comparison of the 2-D-NWT computations to laboratory experiments (0), from [8] and [9] for the case of Fig. 1 without the bottom obstacle, for $x=12.8 \mathrm{~m}$ (i.e., just before the AB). Results show period averaged: (a) surface elevation $\eta$; (b) horizontal velocity $u$ (_ $(-)$ and vertical velocity $w$ $(---)$ at $0.1 \mathrm{~m}$ above the bottom.

drive a 2-D- or 3-D-NS model, with a free surface described by the volume of fluid (VOF) method [18]-[21].

In the NWT, waves can be generated either by a numerical wavemaker, such as used in laboratories, or as actual fully nonlinear waves. An absorbing beach is specified at the other extremity of the NWT, to dissipate incident wave energy before wave overturning and breaking occur [14]. In the NS model, turbulence is represented by large eddy simulation (LES) and sediment transport is computed by a suspended sediment transport model. Although wave computations are 2-D, the NS-LES model is 3-D and implemented over a smaller near-field computational domain than the NWT (e.g., Figs. 3 and 4), where wave-induced suspended sediment transport is calculated. Resolved-scale turbulence, which accounts for a majority of sediment transport, is represented in the 3-D eddies that are simulated in the NS-LES model. Subgrid scale turbulence is represented by an eddy viscosity model. In the sediment transport module, suspended sediment transport is simulated with an advection-diffusion scheme, forced by the wave-induced velocity field from the NS-LES model.

The NWT, NS-LES, and sediment transport models are described in Sections II-A and II-B. This includes a summary of governing equations, boundary conditions, and an overview of numerical methods for each model. In a first application, we simulate the generation of an incident wave group through modulating a periodic wave train and its shoaling over a mild slope. This is to illustrate the capabilities of the NWT to simulate the development of irregular and highly nonlinear waveforms, such as occurs for ocean swells. In a second application, we illustrate our coupled approach with the NWT, NS-LES, and sediment transport models, by generating 2-D periodic waves over a flat bottom and driving sediment transport, in the 3-D near-field NS-LES domain, around a 75\% buried cylindrical object (representing a mine).

Results for the wave fields generated and the sediment transport modeled are discussed in this paper.

\section{BACKGROUND OF NUMERICAL MODELS}

The fully nonlinear 2-D-NWT used in this paper is based on a potential flow approximation, thus assuming irrotational flows of an incompressible, inviscid fluid. Many earlier studies and comparisons with experiments confirm the validity of this model-to-model highly nonlinear wave transformations in coastal areas [7]-[9], [15]-[17]. The 3-D-NS-LES model has been successfully applied to the computation of turbulent boundary layer and separated flows over ripples, induced by oscillatory currents (e.g., [1] and [2]).

Next, we separately summarize the equations of these two models, and of the sediment transport model, and then, explain how the three models were combined to address the problem of sediment transport induced by shoaling waves.

\section{A. Governing Equations and Boundary Conditions}

1) Numerical Wave Tank: Equations for the 2-D-NWT are briefly presented in the following (see [8] and [11]-[14], for details). The velocity potential $\phi(\boldsymbol{x}, t)$ is introduced in the vertical plane $(x, z)$ and the velocity is defined by $\boldsymbol{u}=\boldsymbol{\nabla} \phi=(u, w)$. Continuity equation in the fluid domain $\Omega(t)$ with boundary $\Gamma(t)$ is a Laplace's equation for the potential (Fig. 1)

$$
\nabla^{2} \phi=0 \quad \text { in } \Omega(t)
$$

Using Green's second identity, (1) is transformed into a boundary integral equation (BIE)

$$
\alpha\left(\boldsymbol{x}_{l}\right) \phi\left(\boldsymbol{x}_{l}\right)=\int_{\Gamma}\left\{\frac{\partial \phi}{\partial n} G-\phi \frac{\partial G}{\partial n}\right\} d \Gamma
$$




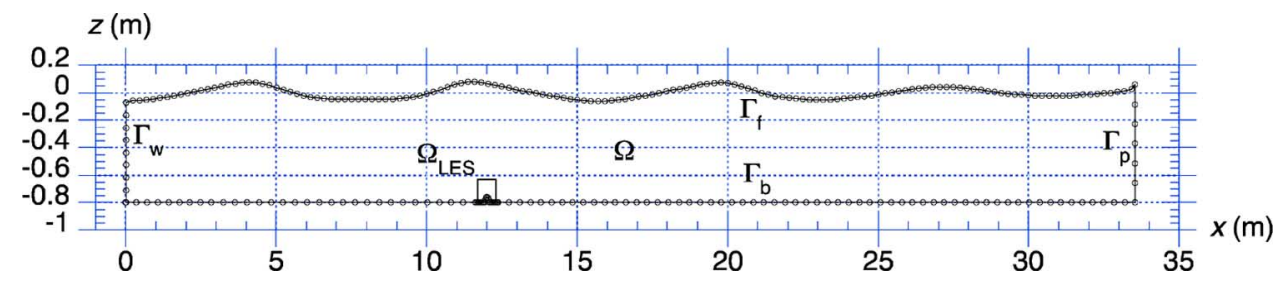

Fig. 3. Typical BEM discretization for a flat bottom case, with a partially buried obstacle, and a periodic wave generation by a piston wavemaker (there is a $5 \times$ vertical exaggeration). Symbol $\Omega_{\text {LES }}$ indicates the location of the submerged NS-LES domain.

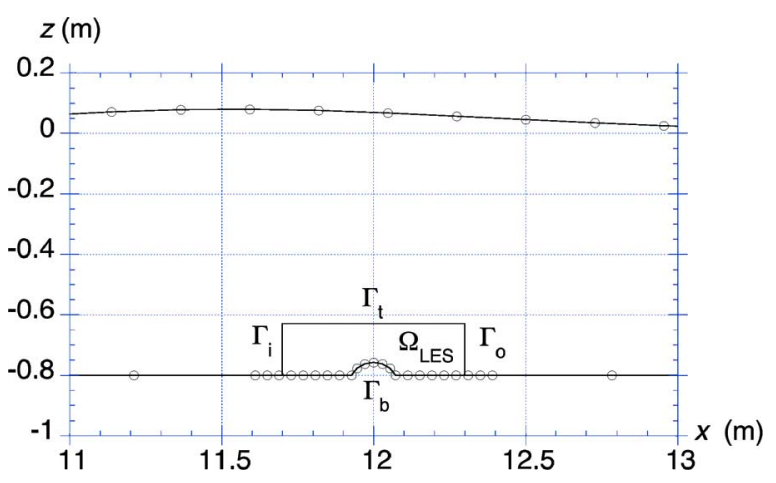

Fig. 4. Closeup of BEM discretization and NS-LES domain with a $75 \%$ buried obstacle. (o) BEM discretization nodes.

where the Green's functions are defined as

$$
\begin{aligned}
G\left(\boldsymbol{x}, \boldsymbol{x}_{l}\right) & =-\frac{1}{2 \pi} \log r \\
\frac{\partial G}{\partial n} & =-\frac{\boldsymbol{r} \cdot \boldsymbol{n}}{2 \pi r^{2}}
\end{aligned}
$$

with $\boldsymbol{r}=\boldsymbol{x}-\boldsymbol{x}_{l}, \boldsymbol{r}=|\boldsymbol{r}|, \boldsymbol{x}=(x, z)$, and $\boldsymbol{x}_{l}=\left(x_{l}, z_{l}\right)$ position vectors for points on the boundary, $\boldsymbol{n}=\left(n_{x}, n_{z}\right)$ the outward unit normal vector to the boundary, and $\alpha\left(\boldsymbol{x}_{l}\right)$ is a geometric coefficient function of the exterior angle of the boundary at $\boldsymbol{x}_{l}$ (usually $\simeq 1 / 2$ for a smooth boundary geometry).

a) Boundary conditions: On the free surface $\Gamma_{f}, \phi$ satisfies the kinematic and dynamic boundary conditions

$$
\begin{array}{ll}
\frac{D \boldsymbol{R}}{D t}=\left(\frac{\partial}{\partial t}+\boldsymbol{u} \cdot \boldsymbol{\nabla}\right) \boldsymbol{R}=\boldsymbol{u}=\boldsymbol{\nabla} \phi & \text { on } \Gamma_{f}(t) \\
\frac{D \phi}{D t}=-g z+\frac{1}{2} \boldsymbol{\nabla} \phi \cdot \nabla \phi-\frac{p_{f}}{\rho} & \text { on } \Gamma_{f}(t)
\end{array}
$$

respectively, with $\boldsymbol{R}$ the position vector on the free surface, $g$ the gravitational acceleration, $z$ the vertical coordinate, $p_{f}$ the free-surface pressure, and $\rho$ the water density. Along the bottom boundary $\Gamma_{b}$, a no-flow condition is prescribed as

$$
\overline{\partial \phi}=0 \quad \text { on } \Gamma_{b}
$$

where the overline denotes specified values.

From Bernoulli equation (5), the wave-induced dynamic pressure within $\Omega$, which will force the NS-LES model, is expressed as

$$
p_{D}=p_{f}-\rho\left\{\frac{\partial \phi}{\partial t}+\frac{1}{2} \boldsymbol{u} \cdot \boldsymbol{u}\right\} .
$$

The computation of the internal solution based on the boundary solution is explicit in the BEM. Details are given as follows.

b) Wave generation: Various methods have been used for wave generation in this NWT. Here, waves are generated on boundary $\Gamma_{w}$, as in laboratory experiments, using a solid piston wavemaker moving according to a prescribed motion $x=x_{w}(t)$. Thus, we have

$$
\frac{\overline{\partial \phi}}{\partial n}=-\frac{\mathrm{d} x_{w}}{\mathrm{~d} t} \quad \text { on } \Gamma_{w}
$$

where the time derivative follows the wavemaker motion. Wavemaker laws are given in the application section. (Note that the piston motion is usually ramped-up over three representative wave periods, by modulating the stroke using a tanh function of time. See references for details.)

c) Wave absorption: During shoaling of a periodic wave over a slope and before breaking (which, on a mild slope, approximately occurs when the ratio of waveheight over depth $\kappa=H / h \simeq 0.8$ ), wave steepness, and asymmetry (both between trough and crest and between front and rear of each wave; e.g., Fig. 1) continuously increase. This paper focuses on sediment transport induced by such highly nonlinear and asymmetric waves, before breaking. Breaking is prevented in the NWT by dissipating incident wave energy in a numerical absorbing beach $(\mathrm{AB})$, located beyond the area of interest (e.g., Fig. 1).

Following [14], the AB is specified for $x \geq x_{a}$ (Fig. 1). In the $\mathrm{AB}$, an external free-surface pressure $p_{f}=p_{a}$ is applied to the free surface, by way of the dynamic free-surface condition (5) (with $z=\eta$ ), to create a negative work and absorb wave energy. Additional wave reduction is induced by causing waves to deshoal, using a bottom geometry within the $\mathrm{AB}$ somewhat similar to a natural bar, with a depth increasing from $h\left(x_{r}\right)$ to $h_{1}$. The AB "absorbing pressure" is specified proportional to the normal particle velocity on the free surface

$$
p_{a}(x, \eta, t)=\nu_{a}(x) \frac{\partial \phi}{\partial n}(\eta(x, t))
$$

where $\nu_{a}$ is the $\mathrm{AB}$ absorption function. This function is first ramped-up from 0 to $\nu_{a o}$, and then, maintained constant over the AB. More specifically

$$
\nu_{a}(x)=\nu_{a \circ} \rho \sqrt{g h_{1}} f(x)
$$

where $\nu_{a o}$ is a nondimensional beach absorption coefficient, and $f(x)$ follows a tanh variation for $x_{a} \leq x \leq x_{r}$ and $f(x)=1$ for $x_{r}<x \leq x_{p}$. 
An absorbing piston (AP; [14]) is also specified at the extremity of the $\mathrm{AB}$, for $x=x_{p}$. This AP moves proportionally to the hydrodynamic pressure force caused by waves, calculated as a function of time in the NWT (see references for details). Grilli and Horrillo [14] showed that the combination AB/AP effectively absorbs incident wave energy in the NWT to within any specified small fraction.

d) Bottom friction: For short distances of propagation over a smooth bottom, wave damping due to bottom friction is quite small, and is usually neglected in numerical models, even for long waves, when the computational domain only spans a few wavelengths (e.g., [15] and [17]). To simulate wave shoaling over a rippled sandy bed, which may induce more significant wave damping due to bed roughness, Grilli et al. [8] simulated an energy dissipation term due to bottom friction in the NWT. They did so by specifying a surface pressure distribution, similar to that detailed previously for the $\mathrm{AB}$, over the shoaling region for $x_{w}<x \leq x_{a}$ (Fig. 1).

For the initial applications reported here, we did not implement a moving sediment bed and, hence, neglected bottom friction effects on wave shape and velocities. Such effects could, however, be included in a later time. More details regarding bottom friction can be found in [9].

e) Solution for internal fields: At each time step, the NWT provides the solution for the velocity potential $\phi$, its normal derivative $\partial \phi / \partial n$, and the time derivative of these [ $(\partial \phi / \partial t)$ and $\left.\left(\partial^{2} \phi / \partial t \partial n\right)\right]$, along-boundary $\Gamma$. Potential $\phi$, for any point $\boldsymbol{x}_{l}$ within domain $\Omega$, can then be explicitly calculated based on the boundary solution, based on BIE (2), (3), expressed for $\alpha_{l}=1$.

Internal velocity fields are required to initialize the NS-LES model, and velocities together with the time derivative of the potential are needed to calculate the wave-induced internal dynamic pressure $p_{D}$ (7). If we denote by $\nabla_{l} \equiv\left(\partial / \partial x_{l}, \partial / \partial z_{l}\right)$ the gradient operator with respect to the coordinates of point $x_{l} \in \Omega$, (2) yields

$$
\frac{\partial \phi}{\partial t}\left(\boldsymbol{x}_{l}\right)=\int_{\Gamma}\left\{\frac{\partial^{2} \phi}{\partial t \partial n} G-\frac{\partial \phi}{\partial t} \frac{\partial G}{\partial n}\right\} d \Gamma
$$

and

$$
\boldsymbol{u}\left(\boldsymbol{x}_{l}\right)=\int_{\Gamma}\left\{\frac{\partial \phi}{\partial n}\left(\nabla_{l} G\right)-\phi\left(\nabla_{l} \frac{\partial G}{\partial n}\right)\right\} d \Gamma
$$

where

$$
\begin{aligned}
\nabla_{l} G & =\frac{\boldsymbol{r}}{2 \pi r^{2}} \\
\nabla_{l}\left(\frac{\partial G}{\partial n}\right) & =\frac{1}{2 \pi r^{2}}\left\{\boldsymbol{n}-2 \boldsymbol{r} \frac{\boldsymbol{r} \cdot \boldsymbol{n}}{r^{2}}\right\} .
\end{aligned}
$$

(Note that the derivatives with respect to internal point coordinates have been directly applied to the Green's functions in the integrand.)

Similarly, internal dynamic pressure gradient fields, which will be used to force the NS-LES model as a function of time, are obtained as

$$
\nabla_{l} p_{D}\left(\boldsymbol{x}_{l}\right)=-\rho\left\{\frac{\partial \boldsymbol{u}}{\partial t}+\boldsymbol{u} \cdot \nabla_{l} \boldsymbol{u}\right\}
$$

where the first term in the right-hand side of (14) is obtained by expressing (12) for $(\partial \phi / \partial t)$ and $\left(\partial^{2} \phi / \partial t \partial n\right)$, and the last term, by applying $\nabla_{l}$ to (12), which yields

$$
\nabla_{\boldsymbol{l}_{i}} u_{j}\left(\boldsymbol{x}_{l}\right)=\int_{\Gamma}\left\{\frac{\partial \phi}{\partial n}\left(\nabla_{l_{i}} \nabla_{l_{j}} G\right)-\phi\left(\nabla_{l_{i}} \nabla_{l_{j}} \frac{\partial G}{\partial n}\right)\right\} d \Gamma
$$

where

$$
\begin{aligned}
\nabla_{l_{i}} \nabla_{l_{j}} G & =\frac{1}{2 \pi r^{2}}\left\{2 \frac{r_{i} r_{j}}{r^{2}}-\delta_{i j}\right\} \\
\nabla_{l_{i}} \nabla_{l_{j}}\left(\frac{\partial G}{\partial n}\right) & =\frac{1}{\pi r^{4}}\left\{n_{i} r_{j}+n_{j} r_{i}+\left(r_{k} n_{k}\right)\left(\delta_{i j}-4 \frac{r_{i} r_{j}}{r^{2}}\right)\right\}
\end{aligned}
$$

in which index notation has been used for convenience, with $i, j, k=1,2$ corresponding to directions $x$ and $z$ for 2-D problems and $\delta_{i j}$ the Kronecker symbol. (Note that, in accordance with Euler equation, the bracket in the right-hand side of (14) is in fact equal to the total flow acceleration at point $x_{l}$ minus gravity.)

2) NS Model: The equations governing fluid motion are continuity and the volume-filtered incompressible NS equations, with the Boussinesq approximation invoked, and a source term for forcing oscillatory or wave-like motion

$$
\begin{aligned}
\frac{\partial \overline{u_{i}}}{\partial x_{i}} & =0 \\
\frac{\partial \overline{u_{i}}}{\partial t}+\frac{\partial}{\partial x_{j}}\left(\overline{u_{i}} \overline{u_{j}}+\frac{\bar{p}}{\rho} \delta_{i j}-\nu \frac{\partial \overline{u_{i}}}{\partial x_{j}}+\tau_{i j}\right) & =-\frac{1}{\rho} \frac{\partial p_{D}}{\partial x_{i}} \\
\tau_{i j} & =\overline{u_{i} u_{j}}-\overline{u_{i}} \overline{u_{j}} .
\end{aligned}
$$

Here, $u_{i}$ is the 3 -D velocity $(i=1,2,3), \tau_{i j}$ is the subfilter scale (SFS) shear stress term, $-\left(\partial p_{D} / \partial x_{i}\right)$ is the momentum source term for the forcing wave motion, corresponding to the dynamic pressure gradient computed in the NWT using (14)-(16), $\nu$ is the kinematic viscosity, $x_{i}$ is the Cartesian coordinate $(i=2$ is vertical), and the overbars denote spatial filtering with a box filter. Here, because of the inherent mismatch between the irrotational NWT and rotational NS solutions, we elected to use one-way coupling by driving the NS solution with the full dynamic pressure from the NWT solution and have not forced the velocities at the NS domain boundary.

The SFS term is represented by the Smagorinsky model, an eddy viscosity model which gives the local eddy viscosity $\nu_{T}$ as proportional to the magnitude of the local strain rate tensor $\left|\overline{S_{i j}}\right|$ [22]. Namely

$$
\begin{aligned}
\tau_{i j}-\frac{\delta_{i j}}{3} \tau_{k k} & =-2 \nu_{T} \bar{S}_{i j} \\
\nu_{T} & =C_{s} \bar{\Delta}^{2}|\bar{S}| \\
|\bar{S}| & =\left(2 \overline{S_{i j} S_{i j}}\right)^{1 / 2} \\
\overline{S_{i j}} & =\frac{1}{2}\left(\frac{\partial \overline{u_{i}}}{\partial x_{j}}+\frac{\partial \overline{u_{j}}}{\partial x_{i}}\right) .
\end{aligned}
$$

Here, $\bar{\Delta}$ is the grid-filter width and $C_{s}$ is a flow-dependent model coefficient; we use $C_{s}=0.021$.

a) Boundary conditions: On the inflow/outflow boundaries $\Gamma_{i}$ and $\Gamma_{o}$, and along the top of our domain $\Gamma_{t}$, we specify 
a gradient-free condition, i.e., $\partial \overline{u_{k}} / \partial x_{1}=0$ and $\partial \overline{u_{k}} / \partial x_{2}=0$, respectively, where $k=1,2,3$ (Figs. 3 and 4).

The bottom boundary condition on $\Gamma_{b}$ is no-slip, or $\overline{u_{i}}=0$, implemented via the immersed boundary method (IBM).

The lateral boundaries are treated as free slip surfaces, satisfying $\partial \overline{u_{3}} / \partial x_{3}=0, \partial \overline{u_{1}} / \partial x_{3}=0$, and $\overline{u_{3}}=0$.

3) Sediment Transport Model: The suspended sediment concentration is modeled with a volume-filtered advection-diffusion equation

$$
\frac{\partial \bar{C}}{\partial t}+\frac{\partial}{\partial x_{j}}\left(\left(\overline{u_{j}}-w_{s} \delta_{i 2}\right) \bar{C}+\chi_{j}\right)=0
$$

where $C=$ sediment concentration by volume fraction and $\chi_{j}=\overline{u_{j} C}-\overline{u_{j}} \bar{C}=$ SFS scalar flux term.

Consistent with the common assumption in the literature that the turbulent Schmidt number $\sigma=1$, the same model is adopted for the SFS terms for both the momentum and sediment concentration equations, i.e., we have used the Smagorinsky model for both.

a) Boundary conditions: For the inflow/outflow and top boundaries $\left(\Gamma_{i}, \Gamma_{o}\right.$, and $\left.\Gamma_{t}\right)$, we have used gradient-free boundary conditions (Fig. 4).

For the bottom boundary $\Gamma_{b}$, we have used an empirical function developed by van Rijn [23] for evaluating the sediment concentration near the bed, as it varies along the bed

$$
\begin{aligned}
C_{a} & =0.015 \frac{d_{50}}{k_{s}} \frac{T^{1.5}}{D_{*}^{0.3}} \\
T & =\frac{u_{*}^{2}-u_{*, \mathrm{CR}}^{2}}{u_{*, C R}^{2}} \\
D_{*} & =d_{50}{\frac{((s-1) g)}{\nu^{2}}}^{1 / 3}
\end{aligned}
$$

where the bottom shear velocity is $u_{*}$, the critical shear velocity is $u_{*, \mathrm{CR}}$, and the roughness height is $k_{s}$.

To compute $u_{*}$, we solved the boundary layer log-law $u / u_{*}=(1 / \kappa) \log \left(y+y_{0}\right) / y_{0}$ for $u_{*}$ at each location along the bed, using as inputs the velocity computed from the previous time step at the grid point nearest to the bed location and the distance of that point to the bed, where $y_{0}=k_{s} / 30$. Nakayama et al. [24] show that, for smooth wall flows, the filtered (in the LES sense) instantaneous wall shear stress and velocity at points above the bed correlate well, and that a log-law relationship is reasonable. Nakayama [25] confirmed that the relationship holds as well for rough walls. For $u_{*, \mathrm{CR}}$, we used van Rijn's representation of the Shields curve as a function of $D_{*}$ [26, eq. (4.1.1)].

Our selection of (22) for predicting the near-bed concentration was based on the following considerations.

- It provides a reference concentration (as opposed to a pickup rate), which was necessary for this first application and use with the IBM (see Section II-B2).

- It has the same form as van Rijn's pickup function [27], employed in the bottom boundary condition in the simulations over ripples performed by Zedler and Street [1], in that it is proportional to $T^{1.5}$. Since van Rijn's pickup function produced physically realistic transport patterns in these simulations, it seemed a reasonable place to start.
- While originally developed to predict the spatially averaged sediment concentration at the top of dunes, it was found to provide the best estimate of concentration at a distance from the bed equal to 5\% of the total depth, as compared with six other formulas tested against data in Garcia et al. [28].

- Although we apply (22) at the bed, rather than at a distance above it, we have reasons to believe (from previous simulations) that its basic form (as proportional to a power of $T$ ) produces physically realistic transport patterns. For quantitative predictions, it will be desirable to introduce a flux-prediction boundary condition, as suggested by Admiraal et al. [29] and used by Zedler and Street [30].

Note, in this paper, we were intent on demonstrating the feasibility of coupling the irrotational wave forcing, the NS and advection solutions, and the IBM of representing the bed. Implementing a flux boundary condition in the IBM is, as noted previously, a nontrivial exercise that is a natural next step beyond this present effort. In addition, Admiraal et al. [29] cite evidence for a phase-lag between the peak shear stress and the sediment concentration at a given cross section. Indeed, that feature of the transport is particularly evident in Zedler and Street [30]. Our formulation estimates the concentration at the bed in proportion to the shear stress (and while we would prefer to estimate the flux there, that was not possible at this time in the evolution of our model), and then, calculates the transport of sediment by advection and turbulent mixing (an LES algorithm) above the bed. Accordingly, based on advection effects alone, sediment concentration peaks above the bed will occur downstream of the shear stress peaks; thus, the phase-lag mechanism is included in our formulation, but the bed boundary condition is not ideal.

\section{B. Numerical Methods Used in Models}

1) Numerical Wave Tank: The BIE (2) is solved by a BEM, by expressing it for $N$ discretization nodes on the boundary $\left(x_{l} ; l=1 \ldots, N\right)$, and defining $M$ higher order elements to interpolate in between discretization nodes. In the present applications, quadratic isoparametric elements are used on lateral and bottom boundaries, and cubic elements ensuring continuity of the boundary slope are used on the free surface. In these elements, referred to as midcubic interpolation (MII) elements, both geometry and field variables are interpolated between each pair of nodes, using the midsection of a four-node "sliding" isoparametric element. The Green's function and its normal derivative in (3) are singular as $r \rightarrow 0$. These singularities require additional treatments whose detailed derivations will not be included here. These can be found in [11] and [13] for both isoparametric and MII elements. It should be noted that, when applying (2) to points $\boldsymbol{x}_{l}$ that are very close to a different part of the boundary (e.g., near corners in Fig. 1), almost-singular integrals will occur when $r$ is very small. The numerical accuracy of such integrals is improved by applying an adaptive integration method.

a) Time integration: Free-surface boundary conditions (4) and (5) are time integrated based on two second-order Taylor 
series expansions expressed in terms of a time step $\Delta t$ and of the Lagrangian time derivative $D / D t$, for $\boldsymbol{R}$ and $\phi$

$$
\begin{aligned}
& \overline{\boldsymbol{R}}(t+\Delta t)=\boldsymbol{R}(t)+\Delta t \frac{D \boldsymbol{R}}{D t}(t)+\frac{(\Delta t)^{2}}{2} \frac{D^{2} \boldsymbol{R}}{D t^{2}}(t)+\mathcal{O}\left[(\Delta t)^{3}\right] \\
& \bar{\phi}(t+\Delta t)=\phi(t)+\Delta t \frac{D \phi}{D t}(t)+\frac{(\Delta t)^{2}}{2} \frac{D^{2} \phi}{D t^{2}}(t)+\mathcal{O}\left[(\Delta t)^{3}\right]
\end{aligned}
$$

respectively. First-order coefficients in the series correspond to free-surface conditions (4) and (5), in which $\phi$ and $\partial \phi / \partial n$ are obtained from the BEM solution of the BIE (2) for $(\phi, \partial \phi / \partial n)$ at time $t$. Second-order coefficients are expressed as $D / D t$ of (4) and (5), and are calculated using the solution of a second similar BIE for $\left(\partial \phi / \partial t, \partial^{2} \phi / \partial t \partial n\right)$, for which boundary conditions are obtained from the solution of the first BIE and the time derivative of (5)-(8). Detailed expressions for the Taylor series coefficients can be found in [11].

At each time step, the overall numerical accuracy is estimated by computing errors in volume and energy of the computational domain. Earlier work showed that these errors are function of both the size (i.e., distance between nodes) and the degree (i.e., quadratic, cubic, etc.) of boundary elements used in the spatial discretization, and of the size of the selected time step [12], [13]. Thus, the optimal time step is adaptively selected based on a mesh Courant number, whose optimal value for MII elements was shown to be about 0.45 . This value is used in the present applications.

In computations involving finite amplitude waves, mean drift currents occur ("Stokes drift"), which continuously move freesurface discretization nodes/Lagrangian markers away from the wavemaker into the NWT. Hence, when performing computations over many wave periods, nodes are redistributed (i.e., regridded at constant arclength intervals over the free surface) after fixed numbers of time steps.

b) Solution for internal fields: All of the integrals in (11), (12), and (15) expressing interior fields are nonsingular and can be evaluated by Gauss quadrature. However, for interior points located very close to boundary $\Gamma$, similarly to corner points in the original discretized BIE (2) (see [12]), the integral kernels will rapidly grow as $r$ becomes small, yielding inaccurate numerical integration over parts of the boundary nearest those points. This is even more so when the operator $\nabla_{l}$ has been applied to the Green's functions, as in (13) and (16), where terms are $O(1 / r)$ to $O\left(1 / r^{3}\right)$ for small $r$. Grilli and Svendsen [12] and Grilli and Subramanya [31] studied these numerical integrations in detail, referred to as quasi-singular integrals (QSI). Following their method, in NWT computations, a geometrical analysis of the discretization is made at each time step, where QSIs are identified based on a distance criterion. During numerical integration of the various BIEs, both for the boundary solution and for internal points, QSIs are then integrated using an adaptive integration method, in which elements are subdivided into an increasingly large number of subsegments, as a function of a distance-based algorithm. The originally selected number of Gauss points is used within each subsegment. Grilli and Subramanya [31] showed that, given enough subsegments, this method can provide almost arbitrary numerical accuracy of the QSIs.

2) NS Model: QUICK [32] is used for the spatial discretization of the advection terms in the momentum (19), and central differences for all other terms. The same methods are used for discretizing the advection-diffusion (21) for the sediment concentration field, except that simple high-accuracy resolution program (SHARP) [33] is used in place of quadratic upstream interpolation for convective kinematics (QUICK) for its improved handling of sharp gradients. Both equations employ a Crank-Nicolson averaging for the diagonal viscous terms, and the Adams-Bashforth method for all other terms, which yields a semi-implicit, quasi-tridiagonal system of equations.

This system of equations is solved with a fractional step method. In this method, an estimate of the velocity field is first obtained by solving NS equations without the pressure term, the pressure Poisson equation is solved for the pressure as driven by the estimated velocity field, and the velocity estimate is corrected with the pressure to satisfy continuity.

Further details about the equations, discretization, and numerical methods can be found in [34] and [35].

a) IBM: Of the many versions of the code originally developed by Zang et al. [34], we employ a modified version of the Tseng and Ferziger [35] code, which includes implementation of the IBM via a ghost cell method [35].

The IBM works by applying the force necessary at the location of the immersed boundary to enforce the physical boundary condition, $u_{i}=0$ in this case. (The implementation of a log-law in the IBM over a complex boundary is exceedingly difficult [36] so, as an approximation, we have assumed that the boundary lies at the $y_{0}$ location where the log-law velocity is zero.) In the ghost point method, this involves providing a description of the immersed boundary shape, identifying the nearest neighbor grid points below (the ghost cells) and above (the interpolating points) the immersed boundary, and using interpolation methods to enforce the boundary condition at the immersed boundary. Values at the ghost point are then set by extrapolation with the interpolation function. Essentially, this amounts to translating the boundary conditions to the location of the physical immersed boundary.

In this case, we fit a vertical second-order Lagrange polynomial function to the values at the two points above a ghost cell.

\section{APPLICATIONS}

Applications presented here include the following two cases:

1) the simulation of shoaling wave groups in the NWT (Figs. 6 and 7);

2) the simulation of periodic wave flows in the NS-LES model and induced suspended sediment transport, over a $75 \%$ buried cylindrical obstacle on the bottom, representing a mine (Figs. 3-5 and 8-10).

We present the first application (case 1) to illustrate the capabilities of the NWT to simulate the shoaling of complex fully nonlinear wave trains. In case 2 , results are qualitatively compared with those of [1] and [2], obtained for the forcing of the NS-LES and sediment transport models over a rippled bed by a simple oscillatory flow. 


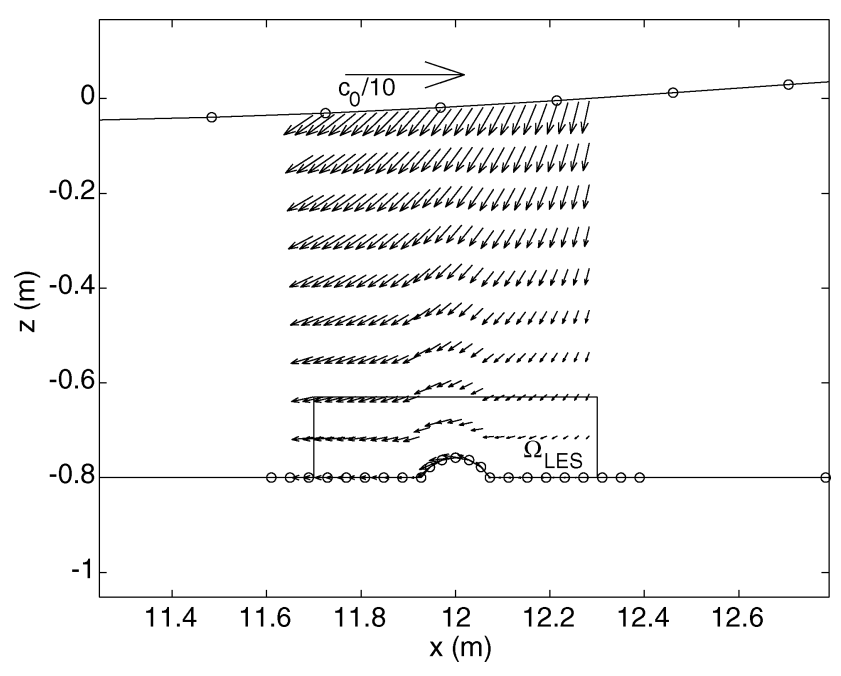

Fig. 5. Example of internal velocity field computed in the 2-D-NWT (at time $t=20.03 \mathrm{~s}$ after the start of simulations), within a vertical cross section in the 3-D NS-LES domain, in a geometry similar to that of Figs. 3 and 4, with a $75 \%$ buried cylindrical obstacle on the bottom. Note, for clarity, the number of internal points has been reduced by a factor of $6 ; c_{o}$ is the theoretical linear wave celerity at depth $h_{o}$.

\section{A. Discretization and Parameters}

1) Numerical Wave Tank: Table I gives a summary of wave and BEM discretization parameters used in the NWT for each application presented here. More details are given later.

a) Discretization: Typical free-surface discretizations (on $\Gamma_{f}$ ) usually have at least 20 nodes per dominant wavelength (e.g., Fig. 3). Lateral boundaries $\left(\Gamma_{w}\right.$ and $\left.\Gamma_{p}\right)$ are typically discretized with only 7-11 nodes. Bottom discretizations (on $\Gamma_{b}$ ) are usually a little coarser than on the free surface, but horizontal node spacing $(\Delta x)$ is reduced on the bottom under the NS-LES domain $\Omega_{\mathrm{LES}}$, to further increase the accuracy of the integrals used to compute internal fields in the BEM solution (Figs. 3-5). Adaptive integrations, with up to $2^{4}$ subdivisions, are performed within four nodes of any corner of the BEM domain, and for all elements located directly under $\Omega_{\mathrm{LES}}$, where points at which internal velocities and pressure gradients are calculated and used for model coupling are specified (Fig. 5). Initial time steps are selected based on the free-surface node spacing, to satisfy the optimal mesh Courant number. Time step is subsequently automatically adjusted as a function of the minimum distance between nodes on the free surface to satisfy the Courant condition. Typical central processing unit (CPU) times in the NWT are less than one second per time step on a desktop computer.

b) Dissipation: In these applications, the NWT was run without bottom friction, and with no regridding applied on the free surface, because no long term computations were run in the BEM model at this stage, as only one single representative period of wave forcing was used to force the NS-LES domain. Longer term computations, for instance with moving bottom (such as migrating ripples), would require regridding the free surface (see [8] and [9]). An absorbing beach AB and an absorbing piston AP (Fig. 1) were specified in each case, to damp incident wave energy and eliminate wave reflection at the NWT extremity.
TABLE I

NONDIMENSIONAL WAVE AND NWT PARAMETERS IN APPLICATIONS; $\omega=2 \pi / T$, WHERE $T$ IS PERIOD; $h_{o}=0.80 \mathrm{~m}$ FOR FLAT BOTTOM OR $2.62 \mathrm{~m}$ FOR WAVE GROUPS DENOTE THE REFERENCE CONSTANT DEPTH (FIg. 1 ); $g=9.81 \mathrm{~m} / \mathrm{s}^{2}$ Is AsSUMED

\begin{tabular}{|l|l|l|l|}
\hline Parameter & Variable & Case & Value \\
\hline \hline Wave & $\omega \sqrt{h_{\circ} / g}$ & Periodic Wave & 0.61 \\
Angular Frequency & & Wave Groups & $1.10,1.26$ \\
\hline Nb. Boundary Nodes & $\mathrm{N}$ & Periodic Wave & 266 \\
& & Wave Groups & 344 \\
\hline Maximum & $u_{p} / \sqrt{g h_{o}}$ & Periodic Wave & 0.054 \\
Paddle Velocity & & Wave Groups & 0.110 \\
\hline Wave Length & $\lambda / h_{\circ}$ & Periodic Wave & 8.75 \\
& & Wave Groups & 3.85 \\
\hline Wave Height & $H / h_{o}$ & Periodic Wave & 0.15 \\
& & Wave Groups & 0.23 \\
\hline
\end{tabular}

2) NS Model: In case 2, the NS-LES model grid dimensions are $0.3 \mathrm{~m}$ long by $0.1727 \mathrm{~m}$ high by $0.0125 \mathrm{~m}$ wide (Fig. 4). For the partially buried obstacle, the bottom is flat in the streamwise direction to either side of a central bump, represented by a $75 \%$ buried cylinder of radius $0.084 \mathrm{~m}$, whose axis runs in the spanwise direction. The NS-LES model is discretized by $82 \times 98 \times 6$ grid points. Hence, we use $82 \times 98=8036$ points in each vertical plane of the 3-D NS-LES domain. The immersed boundary is located above the actual grid boundary so that the total flow domain height is $0.17 \mathrm{~m}$ (discretized by 95 points in the vertical direction over the flat regions). The mine protrudes a vertical distance of $0.042 \mathrm{~m}$ into the flow. The wave forcing is 2-D, as is the bed and, based on our previous work [30], the main structure of the vortices in the vertical plane is not significantly influenced by cross-stream resolution in channel flows. Accordingly, we used only a minimum of cross-stream grid points, assuming that while the flow will not be well resolved in that direction, the results in the vertical plane are not noticeably impacted. No change in the model or setup, however, is required to increase the cross-stream resolution, but the computer time required increases rapidly as one does that.

For the coupled simulations with a periodic wave flow, wave forcing is computed in the NWT, within the fixed embedded NS-LES grid (Figs. 3-5). Thus, the NWT provides the far-field velocities and free-surface wave forcing throughout most of the domain $\Omega$, while the embedded NS solution provides a wellresolved description of the wave boundary layer within domain $\Omega_{\text {LES }}$, with no slip enforced at the bed. The computation of the NS-LES model forcing in the application is further discussed later.

3) Sediment Transport Model: Here, we assume the sediment is sand, with a particle size $d_{50}=200 \mu \mathrm{m}$, a ratio of sediment density to fluid density $s=2.65$ (sediment density is $2650 \mathrm{~kg} / \mathrm{m}^{3}$ ), a roughness height $k_{s}=0.024 \mathrm{~m}$, and a settling velocity $w_{s}=0.026 \mathrm{~m} / \mathrm{s}$, for sediment of this diameter. Because the vertical resolution is not sufficient for quasi-direct NS simulations, and we do assume that the bed is rough, we have used the log-law to estimate the shear stress for use in the sediment transport simulations (see Section II-A3). This has no effect on 


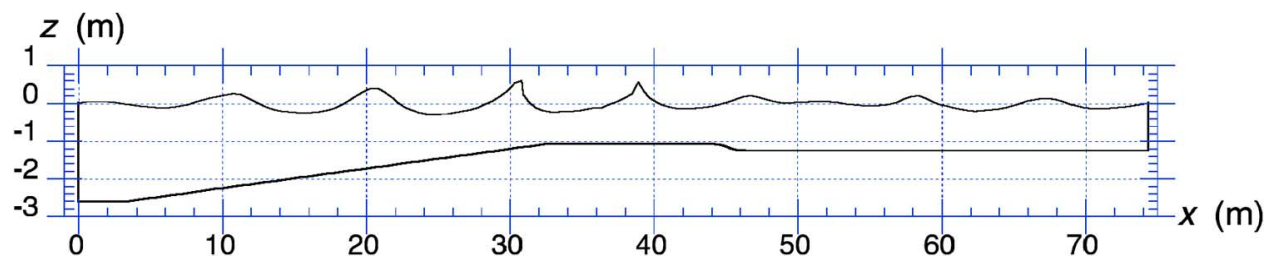

Fig. 6. Case 1: NWT domain used for wave group generation and shoaling over a $1: 20$ slope. The free surface is shown at $t=70 \mathrm{~s}$. The AB is for $x>32 \mathrm{~m}$ and $h_{o}=2.62 \mathrm{~m}$.

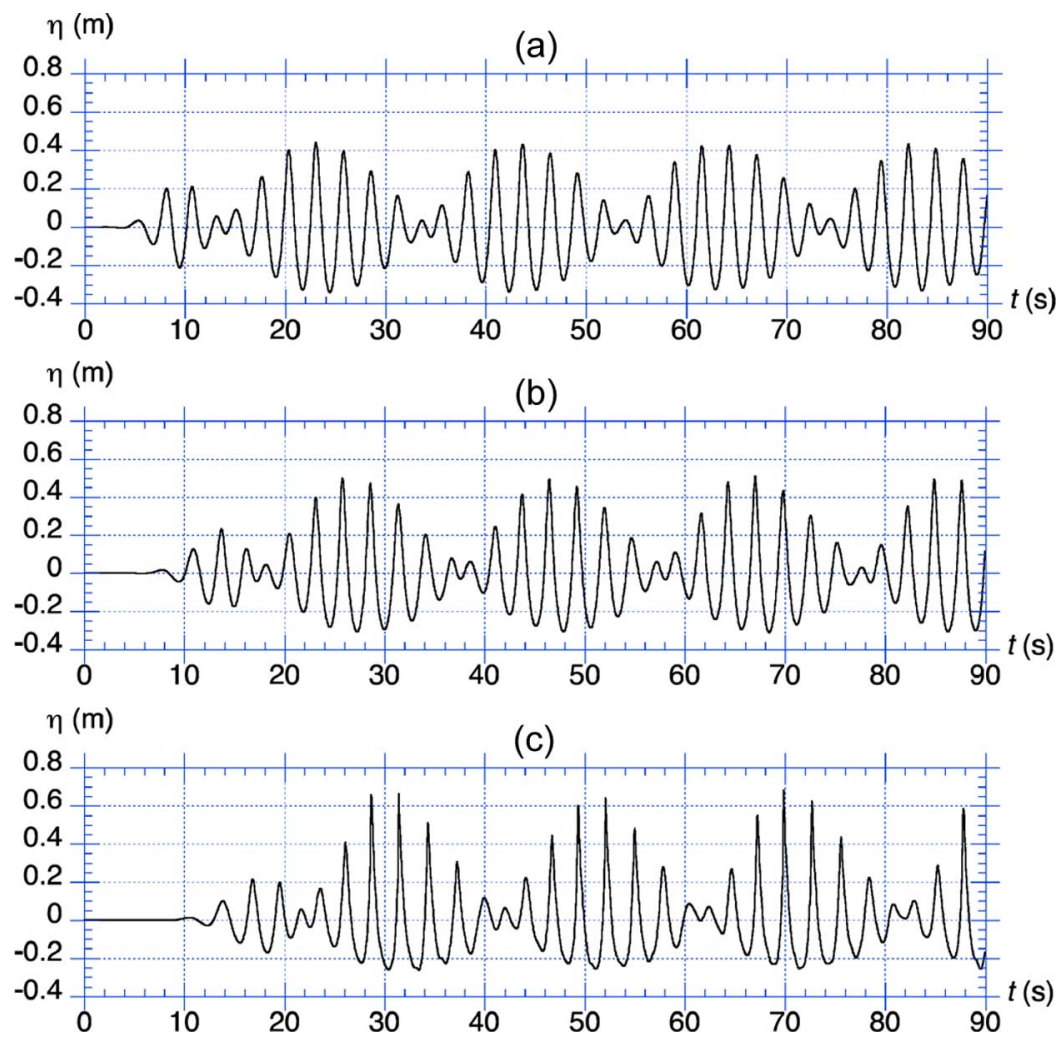

Fig. 7. Case 1: Time series of shoaling wave group (case of Fig. 6), for $x$ being equal to (a) 9.7, (b) 20, and (c) $30 \mathrm{~m}$.

the momentum calculations, but is reasonable as noted in that section.

\section{B. NS-LES Flow Forcing by Internal Fields}

For the NWT-NS-LES-coupled case 2, wave generation is ramped-up over three periods in the NWT and computations are performed until a quasi-steady regime is reached. This usually takes ten wave periods or so. Internal velocity fields are then calculated in the NWT using (12) and (13), to initialize the NS-LES model over a grid of points within the small immersed domain $\Omega_{\text {LES }}$ (e.g., Fig. 5). Pressure gradient fields are calculated over the same grid using (14)-(16), for later time steps, to provide the momentum source term in the NS-LES model (19). Thus, the NWT forcing is applied at each grid point of the NS-LES model, in the horizontal and vertical momentum equations, and varies downstream, vertically, and in time. There is no NWT forcing in the transverse direction for this 2-D flow.

More specifically, in the NWT, to avoid having internal points located very close to the bottom boundary of $\Omega_{\mathrm{LES}}$, which would cause severe QSIs in the calculation of internal fields, requiring time consuming element subdivisions, a smaller number of internal points are used on vertical lines corresponding to the NS-LES grid. These points are placed at equal intervals between the free surface and the bottom of domain $\Omega$ (e.g., Fig. 5). The internal fields computed at these points are then vertically interpolated, using cubic splines, to the exact vertical locations of the NS-LES domain grid points.

Internal fields computed in the 2-D-NWT, for grid points in the vertical plane, are then copied uniformly to each vertical grid in the third dimension of the 3-D NS-LES domain. Initial velocities are thus assumed 2-D when passed from the NWT to the NS-LES model, i.e., with $u_{3}=0$, and for later time steps, internal pressure gradients forcing the NS-LES model are also assumed 2-D, with $\partial p_{D} / \partial x_{3}=0$. Note that, for periodic waves, only a wave period worth of forcing fields is computed and used in a loop to force the NS-LES model computations over many subsequent periods.

\section{Shoaling Wave Groups}

This is Case 1. The NWT wave tank is used to simulate the shoaling of wave groups over a 1:20 slope, starting from an initial depth $h_{o}=2.62 \mathrm{~m}$ (Fig. 1). Fig. 6 shows the domain 

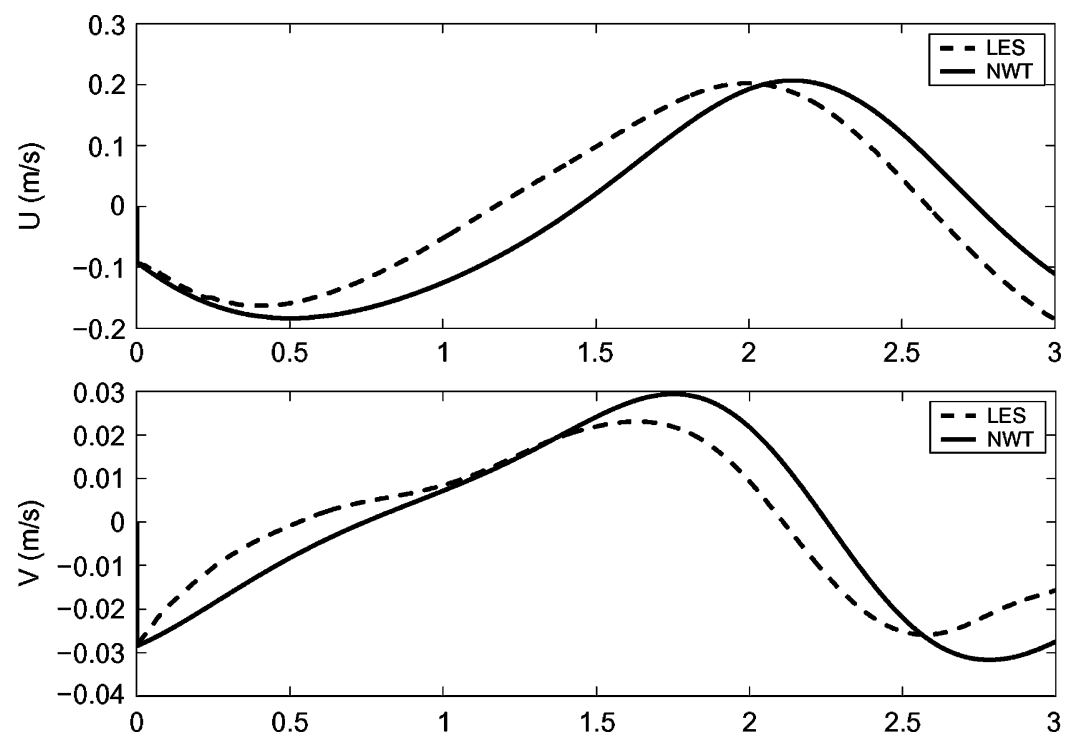

Fig. 8. Case 2: Time series of the horizontal and vertical velocity plotted for the NWT and NS-LES simulations, for the first period of run time, at the same location in the upper left corner of the NS-LES grid ( $x=11.7 \mathrm{~m}$ and $z=-0.63 \mathrm{~m}$ in Fig. 4). The $\mathrm{x}$-axis: time (s).

geometry used for this case. Table I gives general data and parameters. The AB is specified for $x>32 \mathrm{~m}$.

The wavemaker law used for boundary condition (8) for this case is specified as the superposition of two periodic motions of the same amplitude, as $x_{w}=S\left(\sin \omega_{1} t+\sin \omega_{2} t\right)$, with $S=$ $0.27 \mathrm{~m}$ and $\omega_{1}=2.13 \mathrm{rad} / \mathrm{s}$ and $\omega_{2}=2.44 \mathrm{rad} / \mathrm{s}$. This motion is ramped-up over three mean periods based on a tanh law.

Fig. 6 shows the free surface computed at $t=70 \mathrm{~s}$. We see that wave asymmetry and steepness increase as the wave group shoals up the slope, and one wave is close to overturning at $x=$ $31 \mathrm{~m}$. Fig. 7 shows time series of surface elevations for three gages located at $x=9.7,20$, and $30 \mathrm{~m}$. Early in the shoaling, at $x=9.7 \mathrm{~m}$ [Fig. 7(a)], the wave groups are already asymmetric, trough to crest, due to incident wave nonlinearity, with minimum troughs of $0.33 \mathrm{~m}$ and maximum crests of $0.45 \mathrm{~m}$. Nonlinearity and asymmetry of the wave shape further increase for $x=20$ and $30 \mathrm{~m}$ [Fig. 7(b) and (c)], with additional front-to-rear asymmetry at the top of the slope where waveheight reaches more than $90 \%$ the local depth.

It is to be pointed out that, after an initial ramp-up lasting $10 \mathrm{~s}$ or so, all the time series in Fig. 7 show wave groups that recur in time with little change in amplitude, indicating that almost no reflection occurs in the NWT. This confirms that the AB/AP scheme effectively absorbs incident wave energy, as can also be seen in Fig. 6, where waveheight rapidly decreases in the AB, for $x>32 \mathrm{~m}$.

This application clearly shows the ability of the NWT to generate complex, strongly nonlinear, shoaling wave climates, over an arbitrary sloping bottom.

\section{Periodic Wave Flow Over a Cylindrical Obstacle}

This is Case 2. Here, the dynamic pressure gradient calculated in the NWT is used to force the NS-LES model. To verify that this yields realistic results for the flow in the NS-LES model, we plotted the time series for the NWT and NS-LES models, respectively (Fig. 8), at the upper left corner of domain $\Omega_{\mathrm{LES}}$ during the first period of run time ( $x=11.7 \mathrm{~m}$ and $z=-0.63 \mathrm{~m}$ in Fig. 4). We see that velocities in both models agree reasonably well at this time. Later in time, however, this agreement gradually deteriorates (results not shown here), likely for two main reasons. First, waves generated in the NWT are not strictly periodic, due to nonlinearity (e.g., Stokes drift), but in this first illustrative application we force the NS-LES simulations by repeating the NWT data periodically. This causes a slight phase shift, which becomes gradually more pronounced after the initial run period. Second, the magnitudes of the NS-LES simulation results change as these adjust to the formation of the boundary layer over the bottom. For these reasons, the NS-LES simulation results do not match those of the NWT exactly later in time. However, they still produce a realistic periodic wave orbital motion.

Both detailed velocity and sediment concentration results are shown in Figs. 9 and 10, for the 11th period of run time (the first ten periods are model ramp-up). In this case, the suspended sediment concentration field was initialized as a function of the bottom shear stress at the end of the tenth period.

Bedload was not computed for this initial illustration of our coupled model computations. For illustration purpose, we also caused sediment entrainment during most of the flow period, at most locations along the bed, by adjusting upward the value of $k_{s}$.

1) Boundary Layer Dynamics: Fig. 9(a)-(h) depicts the boundary layer development as a series of instantaneous snapshots of the velocity field in a streamwise-vertical plane.

Characteristic of all boundary layers, velocity profiles tend to zero along the bed, with larger near-bed velocities surrounding the crest of the mine. This is consistent with observations that 

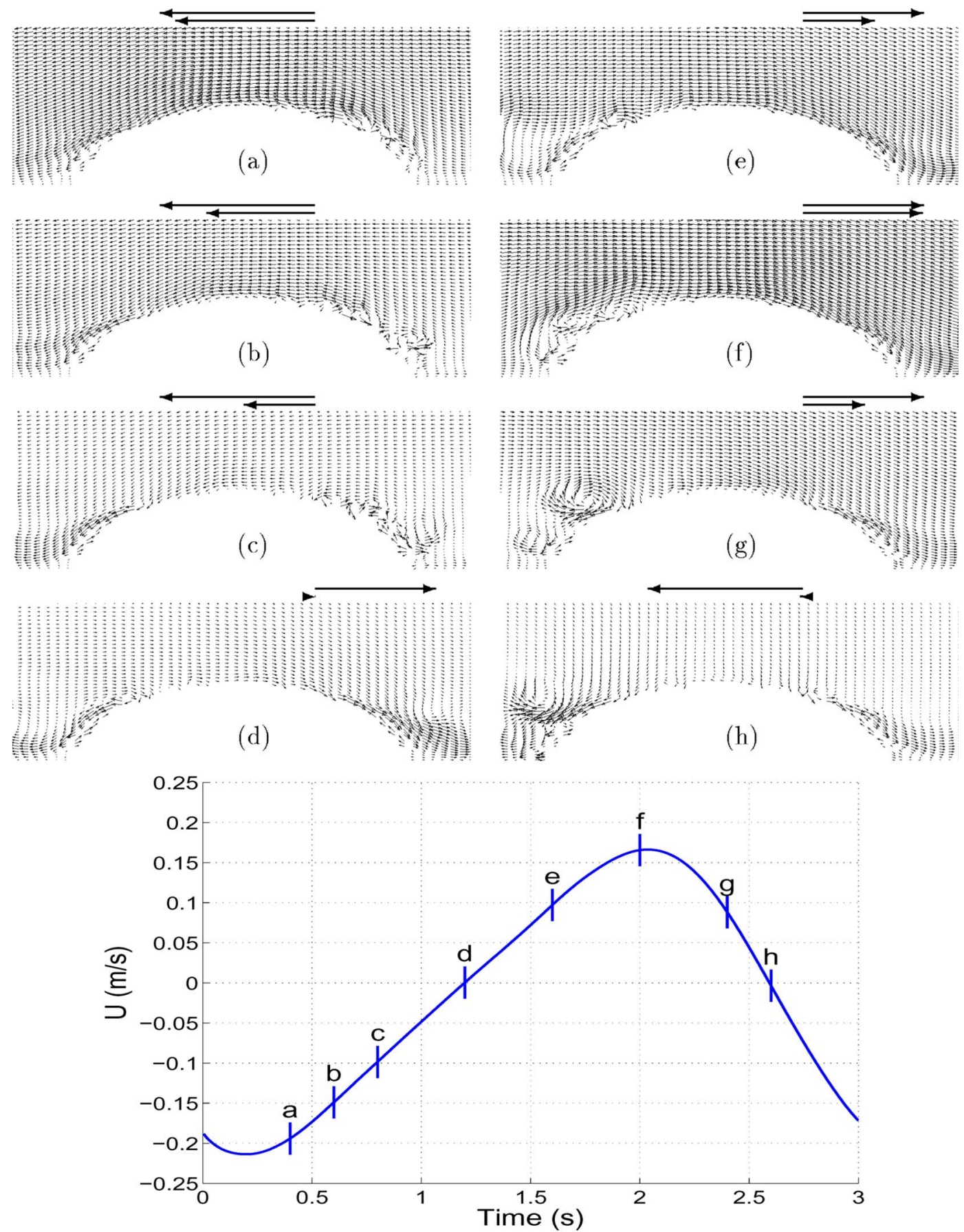

Fig. 9. (a)-(h) Instantaneous plots of the $(u, v)$ velocity field on the centerplane, extending roughly halfway up our numerical domain. The two vectors at the top of each plot indicate the flow direction and magnitude aloft (bottom vector) scaled relative to its maximum value (top vector), on much larger scale than the vectors in the plots. Below: plot of the instantaneous streamwise-averaged $u$ velocity at the top of our domain, with hash marks indicating the timing of vector plots (a)-(h).

the highest shear stresses over ripples occur either just upstream [1] or at the ripple crest [37].

Similar to the findings of [2] for a uniform oscillatory flow over sinusoidal ripples, shear layers form in the lee of the mine during wave phases where the velocity tapers off from its maximum value [Fig. 9(a), (b), and (e)-(g)]. However, the boundary layer development for waves over a $75 \%$ buried cylindrical mine differs significantly from that observed in [2], where an oscillatory flow was forced over sinusoidal ripples. It is thought that the main differences are due to the phasing and spatial distribution of the wave forcing provided by the NWT, as compared to a purely time-dependent oscillatory flow. This is further discussed later.

As the velocity tapers off from its maximum value [Fig. 9(a)-(c) and (e)-(g)], a shear layer first forms on the lee side of the mine [Fig. 9(a), (b), (e), and (f)], as would be expected for a typical oscillatory flow case. As the flow slows enough so that it is about half its maximum value, some of the near-bed velocities reverse [Fig. 9(c) and (g)] and form what looks to be the beginning of a typical lee vortex. However, as the flow slows to zero [Fig. 9(d) and (h)], the pressure gradient distribution on the mine from the NWT acts to intensify the 


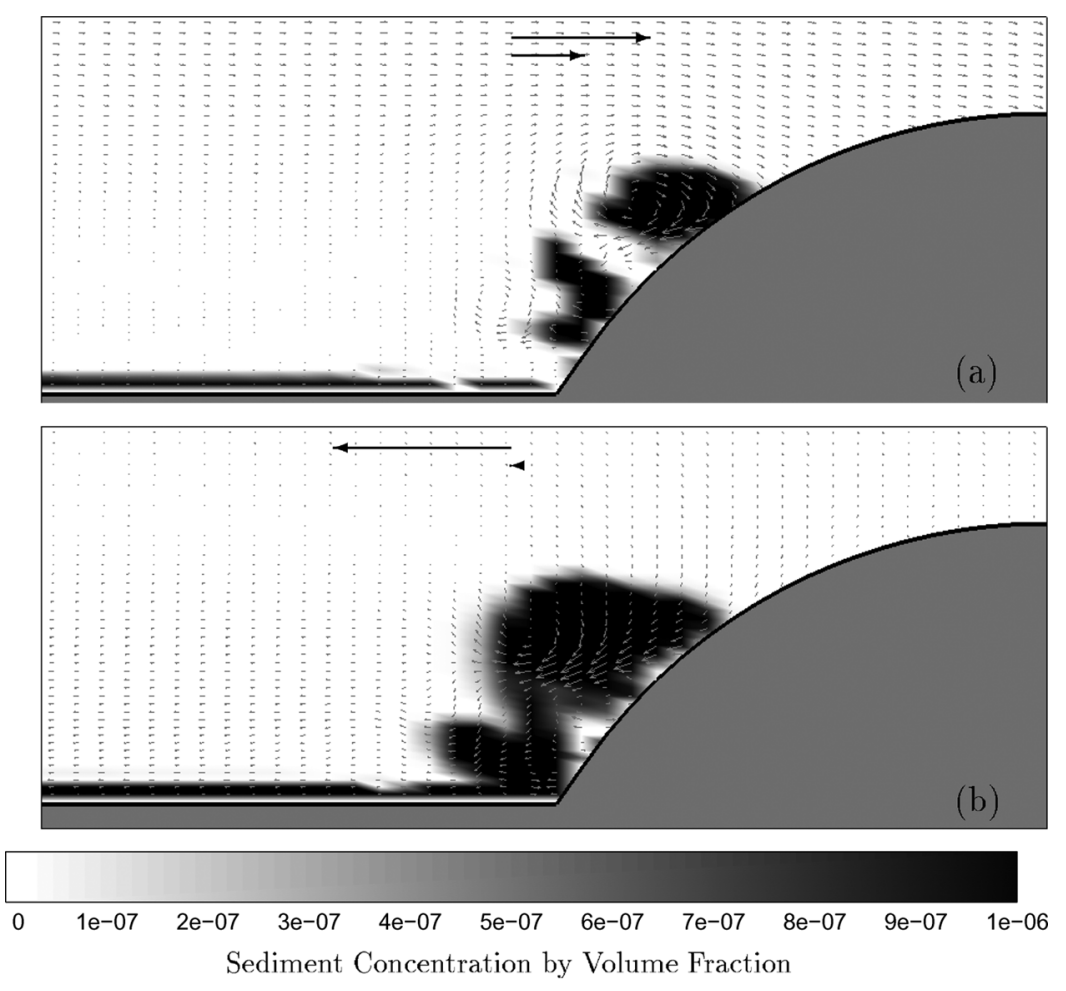

Fig. 10. (a) and (b) Instantaneous plots of sediment concentration contours superimposed on the $(u, v)$, velocity field on the centerplane, extending roughly a third of the way up the numerical domain. The two vectors at the top of each plot indicate the flow direction and magnitude aloft (bottom vector), scaled relative to its maximum value (top vector), on much larger scale than the vectors in the plots. Here, (a) corresponds to Fig. 9(g) and (b) to Fig. 9(h).

flow in the direction down the slope, contrary to the behavior observed in previous simulations of an oscillatory flow over ripples [2].

This jet of fluid is now moving in the direction opposite to the new flow direction. After the flow switches direction, this near-bed jet of fluid, which opposes the new main flow direction, rolls up into a spanwise vortex on the stoss side of the mine [Fig. 9(c) and (g)]. Clearly, the vortex which forms in Fig. 9(g) is much larger and better defined than that in Fig. 9(c). This may be attributed to the asymmetry of the wave (e.g., Fig. 2), because the magnitude of the flow in the "positive" (left to right) direction is considerably weaker than that in the "negative" direction, and, therefore, less capable of destroying the stoss-side vortex/shear layer.

2) Sediment Transport Patterns: As found in [2], the sediment transport patterns follow the flow field very closely. However, the entrainment patterns differ significantly because the boundary condition along the mine enforces the condition $C=0$. Strictly, this is only a first approximation to the correct boundary condition, which would allow for deposition and subsequent pickup on the obstacle boundary $\Gamma_{b}$, as the flux condition of [38, eq. (2)]. For the case studied here, this approximate boundary condition may have contributed to oscillations in the concentration profiles which eventually lead to unrealistic behavior in the sediment concentration field, so that the results presented in this section are taken from the first period after the sediment concentration was initialized. This poor behavior of the $C=0$ boundary condition (applied along the mine) was not expected, since it produced physically reasonable results for a pure-oscillatory case over the mine. We show results during the second half of the 11th flow period, when the flow has had a chance to lose some of the influence of the initial conditions.

In general, sediment is picked up where the shear stresses are greater than critical along the flat bottom regions both upstream and downstream of the mine. It is then oscillated back and forth over the mine due to the action of the flow aloft. This behavior would be rather straightforward if it were not for the formation of vortices on the stoss slope of the mine during every half-period. As in the case of vortex ripples [37], the stoss vortex acts to trap any sediment aloft. Because this vortex forms while the horizontal velocity is large, most of the sediment upstream of the mine (in much lower concentrations) is swept past the vortex during its lifetime, and is trapped there. This process is visualized in Fig. 10. Fig. 10(a) shows the stoss vortex long after its formation, after it has trapped a significant amount of sediment. This sediment trapping may be enhanced by the slightly downward velocity aloft due to the wave coupled with the blocking by the mine. Fig. 10(b) shows that, as the velocity turns around, this puff of sediment is then starting to be swept out of the domain.

\section{CONCLUSION}

The coupling between the NWT and the NS-LES model to simulate wave-induced sediment transport has produced interesting boundary layer dynamics under a realistic orbital wave motion. The main conclusions to be drawn include the following. 
- The approach employed here successfully produces a nonlinear orbital wave-like flow in the NS-LES simulations for periodic waves.

- The boundary layer motions around the partially buried cylindrical obstacle representing a mine, differ considerably between the wave and pure oscillatory flow cases, and as well with those which typically form around ripples in oscillatory flows, as in [2]. The major difference here is that a spanwise vortex forms on the stoss slope of the mine every half period, whereas a spanwise vortex would normally form in the lee of the mine (or ripple) in a pure oscillatory flow case.

- The sediment transport dynamics are very similar to previous simulations with the NS-LES model [1], [2] in that they are heavily guided by the boundary layer motions. Of particular interest, we find that the stoss vortex, which forms every half cycle, acts to trap previously entrained sediment, which flows past it, as the velocity tapers off from its maximum value (cf., [30], which deals with transport over ripples in oscillatory flow and whose entrainment patterns are quite similar to those observed here, albeit with somewhat different timing due to the effect of the obstacle).

The coupled simulations with the NWT, NS-LES, and sediment transport models reported here were only done for a flat bottom with the partially buried obstacle. Cases with a sloping bathymetry are still being worked on, particularly with respect to the NS-LES model convergence, which is slower in such cases, likely due to stronger wave nonlinearity.

The computations of shoaling wave groups illustrate the capabilities of the NWT to simulate the development of irregular and highly nonlinear waveforms, such as occurs for ocean swells. Cases of sediment transport over a sloping bottom, forced by such complex shoaling wave fields will be reported in future work.

Finally, we can think of four model elements that could be added in the future to our coupled model system, namely: 1) implementation of a log-law boundary condition in the momentum equation [36]; 2) a flux boundary condition for suspended sediment transport at the bed, as discussed earlier; 3) a bedload module; and 4) an algorithm to allow dynamic movement of the bed as a function of time (n.b., the current simulations have a fixed bed form). The last algorithm would allow simulation of wave-induced ripple formation and migration [5], or scouring/ burial near-bottom obstacles [6], [10]. Our modeling approach affords this capability and the addition of a moving bottom is ongoing work that will also be reported in future papers.

\section{ACKNOWLEDGMENT}

The authors would like to thank the U.S. Department of Defense (DOD) High Performance Computing Modernization Program for providing a grant of computer time and the anonymous reviewers for the cogent and perceptive comments.

\section{REFERENCES}

[1] E. A. Zedler and R. L. Street, "Large-eddy simulation of sediment transport: Currents over ripples," J. Hydraulic Eng., vol. 127, no. 6, pp. 444-452, 2001.
[2] E. A. Zedler and R. L. Street, "Nearshore sediment transport processes: Unearthed by large-eddy simulation," in Proc. 28th Int. Conf. Coast. Eng., J. M. Smith, Ed., 2002, pp. 2416-2504.

[3] B. Barr, D. Slinn, T. Pierro, and K. Winters, "Modeling unsteady turbulent flows over ripples: Reynolds-averaged Navier-Stokes equations (RANS) versus large-eddy simulation (LES)," J. Geophys. Res., vol. 109, no. C09009, pp. 1-19, 2004.

[4] Y. Chang and A. Scotti, "Numerical simulation of turbulent, oscillatory flow over sand ripples," J. Geophys. Res., vol. 109, no. C09012, pp. $1-16,1991$

[5] S. I. Voropayev, G. B. McEachern, D. L. Boyer, and H. J. S. Fernando, "Dynamics of sand ripples and burial/scouring of cobbles in oscillatory flow," Appl. Ocean Res., vol. 21, no. 5, pp. 249-261, 1999.

[6] S. I. Voropayev, F. Y. Testik, H. J. S. Fernando, and D. L. Boyer, "Burial and scour around short cylinder under progressive shoaling waves," Ocean Eng., vol. 30, no. 13, pp. 1647-1667, 2003.

[7] S. T. Grilli and J. Horrillo, "Shoaling of periodic waves over barredbeaches in a fully nonlinear numerical wave tank," Int. J. Offshore Polar Eng., vol. 9, no. 4, pp. 257-263, Dec. 1999.

[8] S. T. Grilli, S. Voropayev, F. Y. Testik, and H. J. S. Fernando, "Numerical modeling and experiments of wave shoaling over buried cylinders in sandy bottom," in Proc. 13th Offshore Polar Eng. Conf., Honolulu, HI, 2003, pp. 405-412.

[9] S. T. Grilli, S. Voropayev, F. Y. Testik, and H. J. S. Fernando, "Numerical modeling and experiments of periodic waves shoaling over semiburied cylinders in sandy bottom," J. Waterways Port Coast. Ocean Eng., 2007, submitted for publication.

[10] S. I. Voropayev, F. Y. Testik, H. J. S. Fernando, and D. L. Boyer, "Morphodynamics and cobbles behavior in and near the surf zone," Ocean Eng., vol. 30, no. 14, pp. 1741-1764, 2003.

[11] S. T. Grilli, J. Skourup, and I. A. Svendsen, "An efficient boundary element method for nonlinear water waves," Eng. Anal. Boundary Elements, vol. 6, no. 2, pp. 97-107, 1989.

[12] S. T. Grilli and I. A. Svendsen, "Corner problems and global accuracy in the boundary element solution of non-linear wave flows," Eng. Anal. Boundary Elements, vol. 7, no. 4, pp. 178-195, 1990.

[13] S. T. Grilli and R. Subramanya, "Numerical modeling of wave breaking induced by fixed or moving boundaries," Comput. Mech., vol. 17, pp. 374-391, 1996

[14] S. T. Grilli and J. Horrillo, "Numerical generation and absorption of fully nonlinear periodic waves," J. Eng. Mech., vol. 123, no. 10, pp. 1060-1069, 1997.

[15] S. T. Grilli, R. Subramanya, I. A. Svendsen, and J. Veeramony, "Shoaling of solitary waves on plane beaches," J. Waterway Port Coast. Ocean Eng., vol. 120, no. 6, pp. 609-628, 1994.

[16] S. T. Grilli and J. Horrillo, "Fully nonlinear properties of periodic waves shoaling over slopes," in Proc. 25th Int. Conf. Coast. Eng., Orlando, FL, Sep. 1996, pp. 717-730.

[17] S. T. Grilli, I. A. Svendsen, and R. Subramanya, "Breaking criterion and characteristics for solitary waves on slopes," J. Waterway Port Coast. Ocean Eng., vol. 123, no. 3, pp. 102-112, 1997.

[18] S. Guignard, S. T. Grilli, R. Marcer, and V. Rey, "Computation of shoaling and breaking waves in nearshore areas by the coupling of BEM and VOF methods," in Proc. 9th Offshore Polar Eng. Conf., Brest, France, May 1999, vol. III, pp. 304-309.

[19] C. Lachaume, B. Biausser, S. T. Grilli, P. Fraunie, and S. Guignard, "Modeling of breaking and post-breaking waves on slopes by coupling of BEM and VOF methods," in Proc. 13th Offshore Polar Eng. Conf., Honolulu, HI, May 2003, pp. 353-359.

[20] B. Biausser, S. T. Grilli, and P. Fraunie, "Numerical simulations of three-dimensional wave breaking by coupling of a VOF method and a boundary element method," in Proc. 13th Offshore Polar Eng. Conf., Honolulu, HI, May 2003, pp. 333-339.

[21] B. Biausser, S. T. Grilli, P. Fraunie, and R. Marcer, "Numerical analysis of the internal kinematics and dynamics of three-dimensional breaking waves on slopes," Int. J. Offshore Polar Eng., vol. 14, no. 4, pp. 247-256, 2004.

[22] Y. Zang, R. L. Street, and J. R. Koseff, "A dynamic mixed subgridscale model and its application to turbulent recirculating-flows," Phys. Fluids, vol. 5, no. 12, pp. 3186-3196, 1993.

[23] L. C. vanRijn, "Sediment transport 1. Bed-load transport," J. Hydraulic Eng., vol. 110, no. 10, pp. 1431-1456, 1984.

[24] A. Nakayama, H. Noda, and K. Maeda, "Similarity of instantaneous and filtered velocity fields in the near wall region of zero-pressure gradient boundary layer," Fluid Dyn. Res., vol. 35, pp. 299-321, 2004.

[25] A. Nakayama, Personal communication. 2005. 
[26] L. C. vanRijn, Principles of Sediment Transport in Rivers, Estuaries and Coastal Seas. Amsterdam, The Netherlands: Aqua, 1993.

[27] L. C. vanRijn, "Sediment transport 3. Bed forms and alluvial roughness," J. Hydraulic Eng., vol. 110, no. 12, pp. 1733-1754, 1984.

[28] M. Garcia and G. Parker, "Entrainment of bed sediment into suspension," J. Hydraulic Eng., vol. 117, no. 4, pp. 414-435, 1991.

[29] D. M. Admiraal, M. H. Garcia, and J. F. Rodriquez, "Entrainment response of bed sediment to time-varying flows," Water Resources Res., vol. 36 , no. 1 , pp. 335-348, 2000.

[30] E. A. Zedler and R. L. Street, "Sediment transport over ripples in an oscillatory flow," J. Hydraulic Eng., vol. 132, no. 22, pp. 180-193, 2006

[31] S. T. Grilli and R. Subramanya, "Quasi-singular integrations in the modelling of nonlinear water waves," Eng. Anal. Boundary Elements, vol. 13, no. 2, pp. 181-191, 1994.

[32] B. P. Leonard, "A stable and accurate convective modelling procedure based on quadratic upstream interpolation," Comput. Methods Appl. Mech. Eng., vol. 19, no. 1, pp. 59-98, 1979.

[33] B. P. Leonard, "Simple high accuracy resolution program for convective modeling of discontinuities," Int. J. Numer. Meth. Fluids, vol. 8 , pp. 1291-1318, 1988.

[34] Y. Zang, R. L. Street, and J. R. Koseff, "A non-staggered grid, fractional step method for time-dependent incompressible Navier Stokes equations in curvilinear coordinates," J. Comput. Phys., vol. 114, no. 1, pp. 18-33, 1994.

[35] Y. H. Tseng and J. H. Ferziger, "A ghost-cell immersed boundary method for flow in complex geometry," J. Comput. Phys., vol. 192, no. 2, pp. 593-623, Dec. 2003

[36] I. Senocak, A. S. Ackerman, D. E. Stevens, and N. N. Mansour, "Topography modeling in atmospheric flows using the immersed boundary method," Center Turbulence Res., NASA, Stanford, CA, Annual Res. Briefs, 2004.

[37] J. F. A. Sleath, "The suspension of sand by waves," J. Hydraulic Res., vol. 20, no. 5, pp. 439-452, 1982.

[38] I. Celik and W. Rodi, "Suspended sediment transport capacity for open channel flows," J. Hydraulic Eng., vol. 128, no. 12, pp. 1051-1059, 1991.

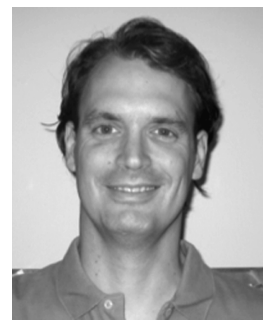

Richard W. Gilbert received the B.S. degree in civil engineering from the University of Arizona, Tempe, and the M.S. degree in ocean engineering from the University of Rhode Island, Narragansett, in ocean engineering in spring 2006

He practiced structural engineering for a few years and obtained his license as a professional engineer. Currently, he works for McLaren Engineering Group, West Nyack, NY, designing coastal structures and performing coastal modeling throughout New England. Study areas include current and wave modeling within harbors, and coastal erosion hazard area mapping and mitigation design.

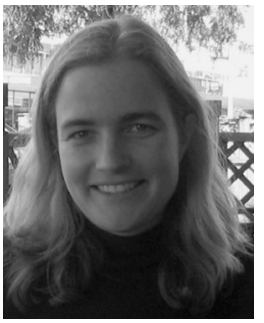

Emily A. Zedler received the B.S. degree in mathematics from the University of California at Davis, and the M.S. degree from the University of California at Berkeley, and the Ph.D. degree from the Stanford University, Palo Alto, CA, in environmental engineering. Her Ph.D. and postdoctoral studies at Stanford University focused on LES of sediment transport over rippled bedforms.

She works at the Santa Clara Valley Water District, San Jose, CA, where she applies her background in environmental engineering to tackle real-world flood control problems. Her work at the District includes open channel flow modeling and sediment transport analysis towards the design of improved capacity and stable channels.

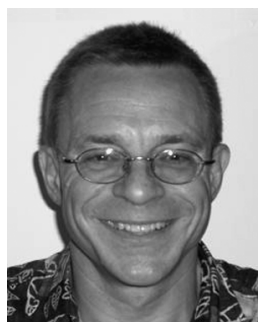

Stéphan T. Grilli received the M.S. and Ph.D. degrees from the University of Liège, Liège, Belgium.

He moved to the United States in 1987. He spent three years as a member of research faculty at the University of Delaware, Newark, before he joined the faculty of the University of Rhode Island, Narragansett, in 1991, where he is now a Distinguished Engineering Professor and Chair of the Department of Ocean Engineering. He studies ocean waves, tsunamis, and related fluid mechanics and marine hydrodynamics problems. Although he conducted experimental work, his research focuses on the numerical modeling of complex nonlinear wave flows, such as wave breaking, extreme wave effects on ocean structures, and wave-induced sediment motion in coastal regions. He also studies waves induced by underwater landslides.

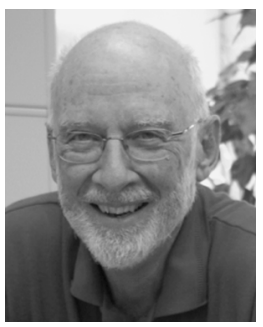

Robert L. Street received the M.S. and Ph.D. degrees from the Stanford University, Palo Alto, CA.

He joined the faculty at Stanford University in 1962. He is the William Alden and Martha Campbell Professor [Emeritus] in the School of Engineering and Emeritus Professor of Fluid Mechanics and Applied Mathematics in the Department of Civil and Environmental Engineering. He studies geophysical fluid motions. His research focuses on the modeling of turbulence in fluid flows, which are often stratified, and includes numerical simulation of coastal upwelling, internal waves and sediment transport in coastal regions, flow in rivers and estuaries, and valley winds in the atmosphere. 